\title{
Design and Implementation of the Temperature Sensor for Health Care Monitoring Based on Optical Fiber Technology
}

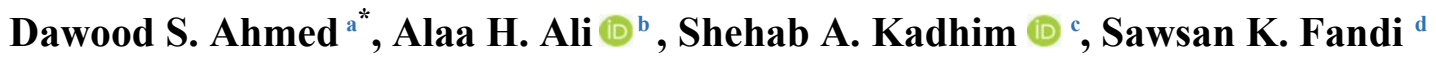 \\ ${ }^{\mathrm{a}, \mathrm{b}}$ Electrical Engineering Dept., University of Technology-Iraq, Alsina'a street,10066 Baghdad, Iraq. \\ ${ }^{c, d}$ Ministry of Science and Technology, Materials Research Directorate, Baghdad, Iraq. \\ *Corresponding author Email: eee.19.51@grad.uotechnology.edu.iq
}

\section{H I G H L I G H T S}

- An optical fiber temperature sensor based on MZI.

- Using a single-mode fiber two different diameters $(125 \mu \mathrm{m}, 60 \mu \mathrm{m})$.

- The sensitivity was good, and the sensitivity is increased about five times when the diameter is decreased.

\section{A R T I C L E I N F O}

\begin{tabular}{l} 
Handling editor: Ivan A. Hashim \\
\hline Keywords: \\
Optical fiber; Temperature sensor; \\
Blood pressure.
\end{tabular}

\begin{abstract}
A B S T R A C T
Human health is represented due to the measurement of vital signs. The basic vital signs are temperature, heart pulse rate, oxygen percentage in blood, blood pressure, etc. These signs are changes according to the physical and mental status of the individual. So measuring and monitoring those signs are very important. In this work, design and implementation of human Temperature Sensor are submitted. This is achieved using optical fiber technology. Two sensor diameters were tested; $125 \mu \mathrm{m}$ and $60 \mu \mathrm{m}$. The obtained results show a shifting in wavelength towards the red region due to temperature application. The submitted sensor has good sensitivity and linearity. Both sensors exhibit good responsivity, sensitivity, and high linearity. The sensitivity is increased about five times when the diameter is decreased.
\end{abstract}

\section{Introduction}

The optical fiber sensors-based nanostructured materials have a large range of applications. Laser light was invented in the year 1960 and after the invention of lasers, researchers showed an interest in the applications of optical fiber sensors. Last few decades, optical fiber sensors played a very important role in the field of sensing technologies. In recent times, many applications have been developed for the detection of different parameters in different areas [1, 2]. Optical fiber sensors (OFS) are devices for measuring strain, temperature, displacement, pressure, electric currents, magnetic fields and various other material and environmental properties. Optical fiber sensors, as well as possessing extreme sensitivity, are electrically passive (which is important for safety in some applications such as medical applications). The general principle behind optical fiber sensors is quite simple. In communication applications of optical fiber, it is undesirable for the light waves being sent through an optical fiber to be affected by the environment, or the message being sent may be corrupted. An optical fiber sensor detects the effect(s) that a selected environmental property has on light being sent through the optical fiber. Therefore, by measuring the changes in some parameters of the light exiting the optical fiber 'sensor', the property of the environment that caused the change in the light can be measured [3]. The optical fiber can actively take part in the sensing mechanism i.e. the optical fiber structure is modified for sensing function. This type of sensor is termed as intrinsic optical fiber sensor. The optical fiber can also serve as a mere light guiding element to an external transducer, this type of sensor is termed as extrinsic optical fiber sensor [4]. Fiber optic sensors are excellent candidates for tracking environmental changes, and they have several of advantages over traditional electronic sensors $[5,6,7]$. Due to their small size and immunity to electromagnetic interference and radio frequency interference, lightweight, and high sensitivity. Fiber optics sensors have many applications compared to traditional sensors, because they have many advantages such as small size, high sensitivity, and immunity to electromagnetic interference such as energy, civil infrastructure, transportation monitoring, and medical application [8].The sensors are biocompatible (non-toxic and biochemically inert) and resistant to electromagnetic interference. They may be used to contact external organs such as the skin or surgically exposed surfaces noninvasively. They may also be inserted through the cavities of the body (endoscopic approach), inserted interstitially by minimally trocars, or placed trocars (e.g., hollow bore needles) intravascular due to their flexibility and thin outer diameter. Consequently, measurements in difficult-to-reach areas of the human body can be carried out with greater "local" sensitivity [9]. 
Below previous studies will be presented on the use of some techniques to measure human temperature.

In 2017, Chunya Wang, et al. the Silk carbon fiber membranes (CFM) structure each sensor is made to be unique insensitive in response to other stimuli, allowing the integrated E-skin to sense temperature and pressure simultaneously. It has been demonstrated that the E-skin can detect and differentiate exhaling, pressing fingers together, temperature and pressure spatial distribution in a way that Single-mode sensors cannot. The temperature sensor has a 0.81 percent per centigrade temperature sensitivity with a gauge factor of 8,350 at 50 percent strain, the strain sensor has an exceptionally high sensitivity, allowing it to detect local strain caused by subtle pressure stimuli [10].

In 2018, Seungyong Han, et al. Small, Sensors that resemble skin and are capable of accurate, indefinite physiological health measurements have a lot of potential in clinical health-care services. The use of sensors dispersed it will be an ideal location for full-body, spatiotemporal visualization of physiological processes a significant step forward in this direction. Temperature and pressure sensors that look Skin-like and are Battery-free and can be used all over the body, materials, interface designs, wireless power delivery and communication methods, as well as overall device architectures [11].

In 2019, Fahimeh Dehkhoda et al. created a temperature sensor based on light emitting diodes (LEDs) that use less space to ensure consistent bias and current measurement by using the LED as its own sensor and a complementary metal oxide semiconductor (CMOS) electronic circuit interface. To accomplish this, a second-generation current conveyor (CCII) configuration is used in this circuit which was implemented in $0.35 \mathrm{~m}$ CMOS technology. The temperature-sensing functionality was tested by interfacing various mini-LEDs in saline tissue models prior to in vivo surgery, and the developed circuits were experimentally characterized. With an operating frequency of up to $130 \mathrm{kHz}$, the outcomes of the experiment demonstrate the versatility the reliability of the CCII-based technique and the efficiency of CMOS electronics achieving a $0.2^{\circ} \mathrm{C}$ resolution for surface temperatures up to $+45^{\circ} \mathrm{C}[12]$.

In 2020, Hsuan-Yu Chen, et al. The use of an infrared thermometer to measure the temperature of the forehead is used to quickly test for fever and identify coronavirus victims (COVID-19). The efficiency of the temperature and this kind of thermometer threshold for fever inspecting are investigated. In both ears, the tympanic temperature, as well as the temperature of the wrists and the top of the head, are measured using two mechanical thermometer IRTs (infrared thermometer) and two medical tympanic IRTs in this study. For the two experiments, the tympanic temperature measured with the BRAUN IRT was $36.9^{\circ} \mathrm{C} 0.286^{\circ} \mathrm{C}$ and $36.937^{\circ} \mathrm{C} 0.301^{\circ} \mathrm{C}$, respectively. For the forehead temperature, the BRAUN measures $34.714^{\circ} \mathrm{C} 0.392^{\circ} \mathrm{C}$ and the THI-301 IRT measures $34.80^{\circ} \mathrm{C} 0.392^{\circ} \mathrm{C}$. For the two experiments, the temperature variations between the tympanic temperature and the forehead temperature are $2.20^{\circ} \mathrm{C} 0.411^{\circ} \mathrm{C}$ and $2.107^{\circ} \mathrm{C} 0.311^{\circ} \mathrm{C}$, respectively. For the two studies, the temperature differential between the tympanic membrane and the forehead ranges between 2.1 and 2.2 degrees Celsius [13].

In 2021, Dong, Wei-Chong MD, et al. Hollow fiber centrifugal ultrafiltration was used to prepare serum samples, the filtrate was directly injected into a ultra-high performance liquid chromatography apparatus (UPLC BEH C18 column $(50 \times$ $2.1 \mathrm{~mm}, 1.7 \mathrm{~m})$ for analysis. At a flow rate of $1.0 \mathrm{~mL} / \mathrm{min}$, the mobile step was made up of acetonitrile and water $(8: 92)$. The temperature of the column was kept constant at $30^{\circ} \mathrm{C}$. The wavelength that was detected was $210 \mathrm{~nm}$. From 0.625 to 80 $\mathrm{mcg} / \mathrm{mL}$, LEV showed a linear relationship $(\mathrm{r} 2=0.999)$. The levetiracetam (LEV) study had a detection limit of 0.125 $\mathrm{mcg} / \mathrm{mL}$. The review was cut down to 4 minutes. The new approach yielded a 96.6 percent -100.1 percent recovery rate for LEV, while the overall recovery rate was 93.2 percent -96.8 percent. The intraday and interday precision relative SD was both 7.3 percent. After three freeze-thaw periods, stability was a must maintained for 24 hours at room temperature, and 21 days at $80^{\circ} \mathrm{C}$ LEV concentrations in the serum of 19 patients were measured using this procedure and was found to be successful [14].

\section{Methodology}

In this work, a description of an optical sensor that measures human temperature had been submitted. A temperature sensor based on the Mach-Zinder interferometer technology, using a Single-mode fiber two different diameters will be tested. The standard diameter of $125 \mu \mathrm{m}$ and sensors with a diameter of $60 \mu \mathrm{m}$. The diameter had been reduced using the chemical etching method.

The Mach-Zehnder interferometer is a particularly simple device for demonstrating interference by division of amplitude. A light beam is first split into two parts by a beamsplitter and then recombined by a second beamsplitter. Depending on the relative phase acquired by the beam along the two paths the second beamsplitter will reflect the beam with efficiency between 0 and $100 \%$. The operation of Mach-Zehnder interferometer is often used as an example in quantum mechanics because it shows a clear path-choice problem [15].

\section{Experimental Work}

To demonstrate the execution ability an optical sensor for measuring human body temperature, a realistic physical model was created to simulate by using water bath. The schematic diagram and experimental setup of the temperature sensor based on optical fiber are shown in Figures (1) and (2).

A temperature sensor based on the Mach-Zinder interferometer technology, using a Single-mode fiber two different diameters will be tested. The standard diameter of $125 \mu \mathrm{m}$ and sensors with a diameter of $60 \mu \mathrm{m}$. The diameter had been reduced using the chemical etching method. Through immersion the middle region of stripped and cleaned Single-mode fiber with hydrofluoric acid (HF) for 40 minutes. The original concentration of the HF acid is $40 \%$. This acid had been diluted using Distilled water (DW) by percentage (2 DW:1 HF) is shown in figure (3). Mach- Zehender interferometry technique is used to design the temperature sensor. Fiber-coupled laser diode source (LPS-FC) having wavelength $1550 \mathrm{~nm}$ and optical power (1.5 $m W$ ) is connected to the input port of 1 X2 Optical Coupler (OC1) with a coupling ratio of 50:50. One of the output ports of the $\mathrm{OC} 1$ is connected to the reference arm of the sensor. This arm is still fixed without any perturbation. The second arm of the $\mathrm{OC} 1$ is connected to the sensor arm of the structure. This arm is immersed in the controlled water bath. The reason behind using the water bath is to insure applying temperature around the sensor fiber. The temperature range is $(35-41)^{\circ} \mathrm{C}$ which is considered the range of human temperature. Then, the output from the reference arm and the sensor arm were collected by 
another $2 \mathrm{X} 1$ optical coupler $\left(\mathrm{OC}_{2}\right)$. The collected spectra then analyzed using an Optical Spectrum Analyzer (OSA- Thorlabs $203)$ with a resolution of $0.1 \mathrm{~nm}$ and a wavelength range (1000-2500) $\mathrm{nm}$.

\section{Result and Discussions}

\subsection{SMF without etching}

The use of Single-mode fiber without etching with diameter $125 \mu \mathrm{m}$ as a temperature sensor to the acquired waveform of strains from the body surfaces. This waveform is pulse waves increasing and decreasing temperature. For laboratory experimental, to simulate temperature conditions at the actual sensor site, a realistic physical model was developed. Figure (4) shows the transmission spectra of the optical signal propagated along the optics sensor.

From the above figure, a shifting in wavelength towards the red region and attenuation in peak power due to the application of temperature.

To test the function of the sensor with increasing and decreasing the temperature first increasing of temperature had been done then a decrease in temperature is applied. As it clear in figure (5), both cases show a good sensitivity and high linearity. The sensitivity was obtained from the linear relationship between the wavelength and temperature. The sensitivity in case of increasing and decreasing temperature is equal to $5 \mathrm{pm} /{ }^{\circ} \mathrm{C}$ and (-) $5.5 \mathrm{pm} /{ }^{\circ} \mathrm{C}$ respectively.

\subsection{SMF with etching}

To enhance the sensor performance, an optical fiber with a diameter $60 \mu \mathrm{m}$ had been used. The same procedure for the standard fiber is done. This waveform is pulse waves increasing and decreasing temperature. Figure (6) shows the transmission spectra of the optical signal propagated along the optics sensor.

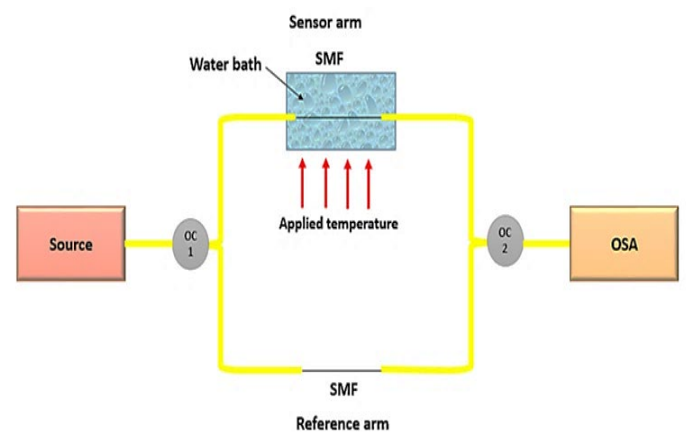

Figure 1: Schematic diagram of temperature sensor based on optical fiber

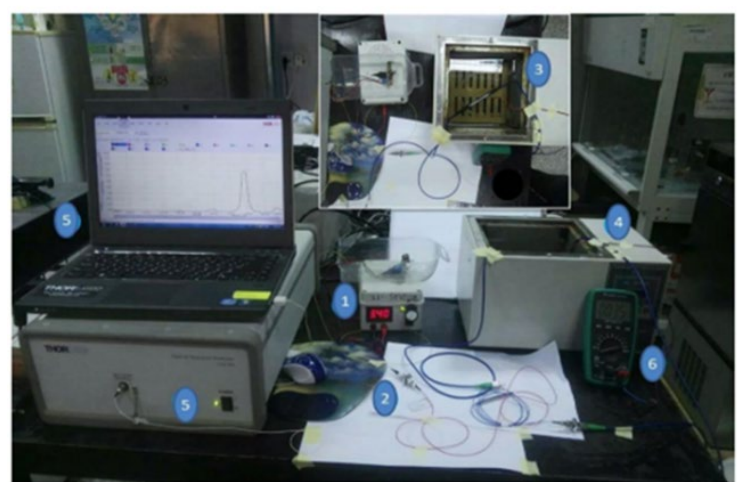

Figure 2: (1) light source, (2) optical circulator, (3) optical fiber, (4) water bath, (5) OSA with PC (6) Digital thermometer

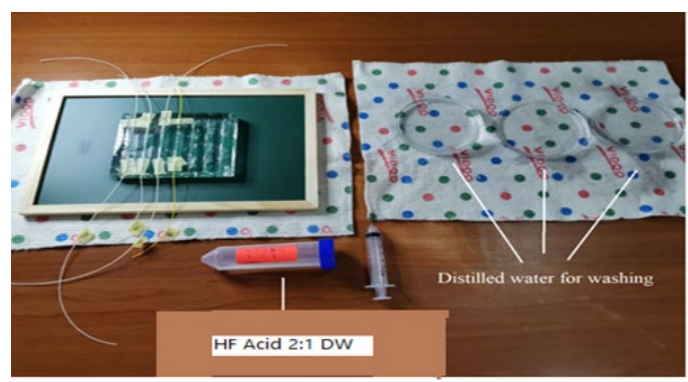

Figure 3: Chemical Etching

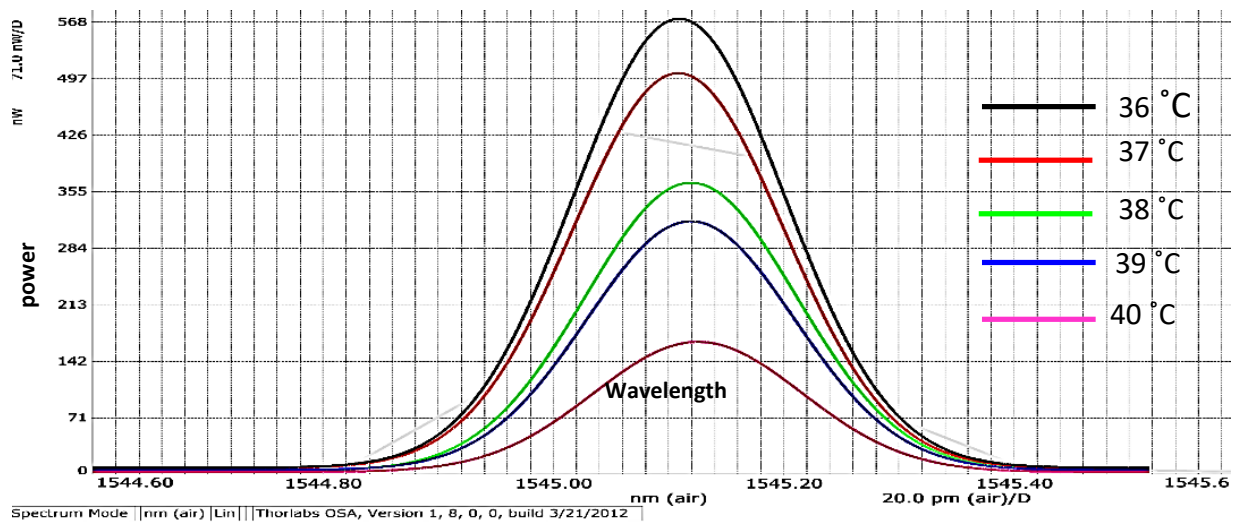

Figure 4: The transmission spectra of the optical signal propagated along with the Singlemode fiber with a diameter $125 \mu \mathrm{m}$ for the temperature sensor 


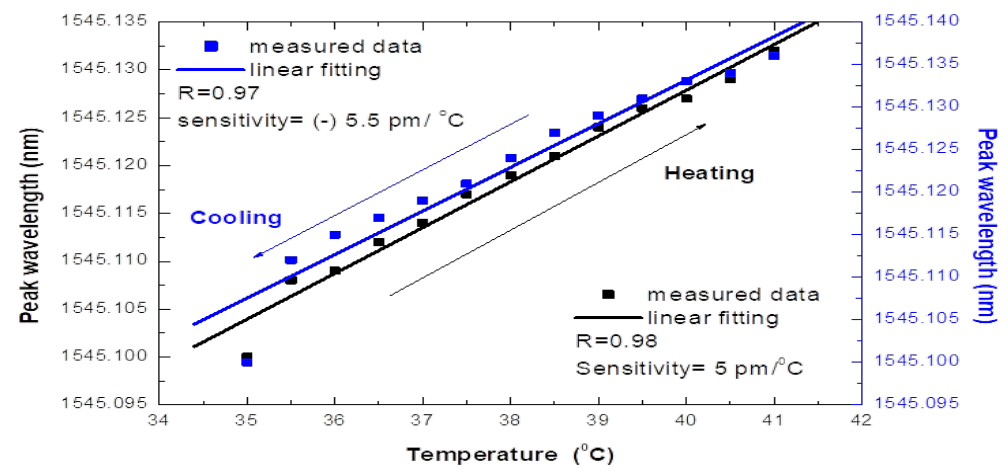

Figure 5: The relation between the shifting in peak wavelength and applied Temperature

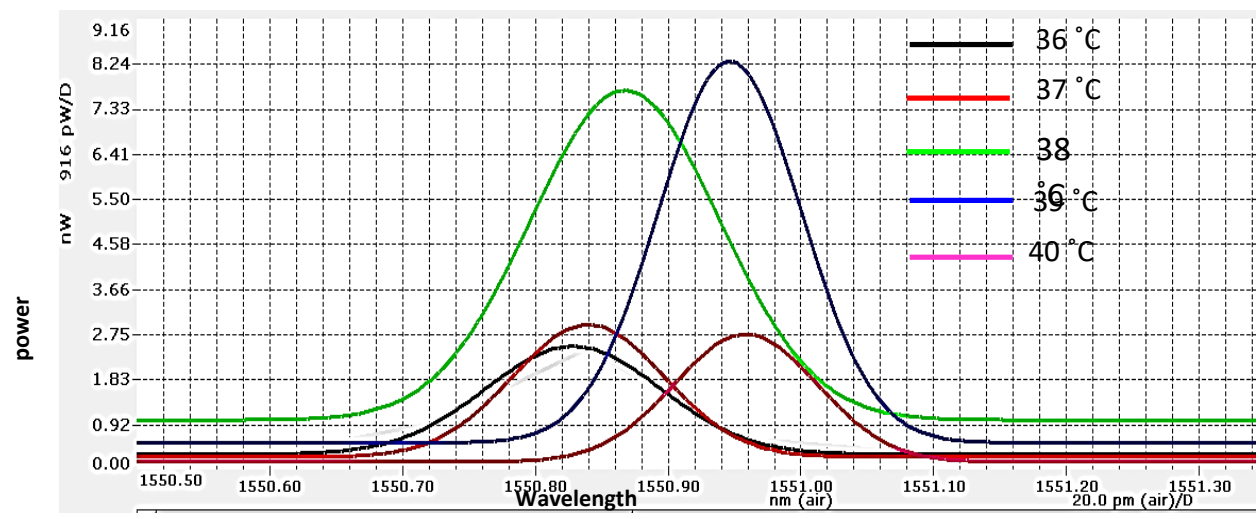

Figure 6: The transmission spectra of the optical signal propagated along with the Singlemode fiber with a diameter $60 \mu \mathrm{m}$ for the temperature sensor

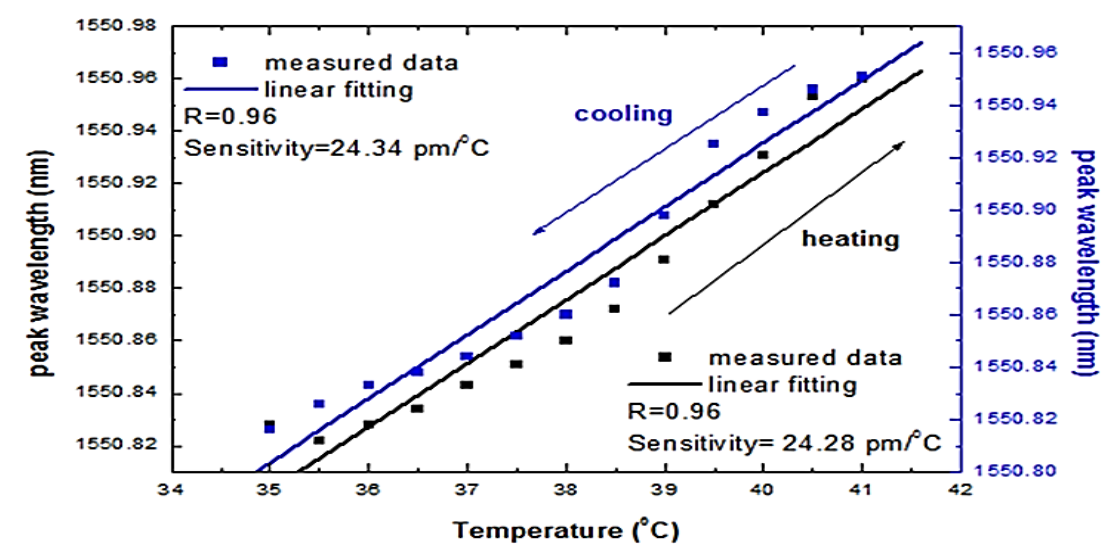

Figure 7: The relation between the shifting in peak wavelength and applied temperature

From the above figure, a shifting in wavelength towards the red region and attenuation in peak power due to the application of temperature.

The relation between the shifting wavelength and temperature illustrated in figure (7), the sensor shows good sensitivity and linearity. The sensitivity was obtained from the linear relationship between the wavelength and temperature. The sensitivity for increasing and decreasing temperature was $24.28^{\circ} \mathrm{pm} / \mathrm{C}$ and $24.34^{\circ} \mathrm{pm} / \mathrm{C}$.

From the above results, it could be noticed that the sensor performance is enhanced due to the decrease in sensor diameter. This is because the reduction of fiber diameter leads to an increase in the evanescent field and thus more light modes will be effected by the outer environment.

\section{Conclusion}

In this work, a description of an optical sensor that measures human temperature had been submitted. Two sensor diameters were tested $125 \mu \mathrm{m}$ and $60 \mu \mathrm{m}$. Both sensors exhibit good responsivity, sensitivity, and high linearity. The sensitivity is increased about five times when the diameter is decreased. Also, the performance of the sensor in the case of increasing and decreasing is linear. The remarkable properties of the optical fiber like small size, lightweight, no toxic effect, and immune to electromagnetic radiation make them a very good choice in different applications like in medical fields.

\section{Author contribution}

All authors contributed equally to this work. 
Funding

This research received no specific grant from any funding agency in the public, commercial, or not-for-profit sectors.

Data availability statement

The data that support the findings of this study are available on request from the corresponding author.

Conflicts of interest

The authors declare that there is no conflict of interest.

\section{References}

[1] L. Zhang, J. Lou and L. Tong, A review of Micro/Nanofiber Optical Sensors, Photonic. Sens., 1(2011) 1-42. https://doi.org/10.1007/s13320-010-0022-Z

[2] A. Urrutia, J. Goicoechea, and F. J. Arregui, A review of Optical Fiber Sensors Based on Nanoparticle-Embedded Coatings, J. Sens., 2015 (2015) 805053. https://doi.org/10.1155/2015/805053

[3] R. Kashyap, Photosensitive optical fibers: devices and applications, Opt. Fiber. Technol., 1 (1994) 17-34. https://doi.org/10.1006/ofte.1994.1003

[4] Narayanaswamy, Ramaier, Wolfbeis, Optical sensors: industrial, environmental and diagnostic applications, Springer, Berlin, (2004).

[5] Fidanboylu, K. and Efendioglu H. S., Fiber Optic Sensors and Their Applications 5th International Advanced Technologies Symposium (IATS'09 Karabuk, Turkey, 2009.

[6] M. S. Shaikh and M. A. Patil, Analysis, Designing and Working Principal of Optical Fiber (Of) Biosensors, Int. J. Eng. Sci., 1 (2012) 52-57.

[7] J. M. López-Higuera, L. R. Cobo, A.Q. Incera, and A. Cobo, Fiber optic sensors in structural health monitoring, J. Light. Technol., 29 (2011) 587-608. https://doi.org/10.1109/jlt.2011.2106479

[8] Novais S., Optical Fiber Sensors for Challenging Media, Ph.D. Thesis, University of Aveiro, Aveiro, Portugal, (2019).

[9] Welch, A.J, V. Gemert, M.JC, Overview of optical and thermal laser-tissue interaction and nomenclature. In: Welch AJ, van Gemert MJC (eds) Optical-thermal response of Laserir radiated tissue, 2nd. Berlin, Springer, (2011). https://doi.org/10.1007/978-90-481-8831-4

[10] C. Wang, K. Xia, M. Zhang, M. Jian, and Y. Zhang, An All Silk-Derived Dual-Mode E-skin for Simultaneous Temperature-Pressure Detection, ACS Appl. Mater. Interfaces, Cite this: ACS Appl. Mater. Interfaces ., 9 (2017) 3948439492. https://doi.org/10.1021/acsami.7b13356

[11] S. Han, J. Kim, S. M. Won, Y. Ma, D. Kang, Z. Xie, K.T. Lee, H. U. Chung, A. Banks, S. Min, S. Y. Heo, C. R. Davies, J.W. Lee, C. H. Lee, B. H. Kim, K. Li, Y. Zhou, C. Wei, X. Feng, Y. Huang, and J. A. Rogers, Battery-free, wireless sensors for full-body pressure and temperature mapping, Sci. Transl. Med., 435 (2018). https://doi.org/ $\underline{\text { 10.1126/scitranslmed.aan4950 }}$

[12] F. Dehkhoda, A. Soltan, N. Ponon, A. O’Neill, A. Jackson, and P. Degenaar, A current-mode system to self-measure temperature on implantable optoelectronics, Biomed. Eng. Online., 18, 117 (2019). https://doi.org/10.1186/s12938-0190736-0

[13] H. Y. Chen, A. Chen and C. Chen, Investigation of the Impact of Infrared Sensors on Core Body Temperature Monitoring by Comparing Measurement Sites, J. Sens., 20 (2020). https://doi.org/10.3390/s20102885

[14] Dong, W-chong,Guo, J-liang, Yang, Xiu-ling, Wang, Shu-mei, Yuan, Ye, Zhao, M-qiang, Jiang, Ye Zhang, Zhi-qing, A Direct Injection Technique to Improve Biosafety to Analyze Levetiracetam Concentrations in Human Serum and Its Application in Therapeutic Drug Monitoring, Therapeutic Drug Monitoring, 43 (2021) 292297. https://doi.org/10.1097/ftd.0000000000000802

[15] K P Zetie, S. F. Adams, R M Tocknell, How does a Mach-Zehnder interferometer work, iopscience.iop.org. 35(2000). https://doi.org/10.1088/0031-9120/35/1/308 